\title{
Neutrino oscillations with the OPERA experiment
}

\author{
Giuliana Galati*i \\ Università degli Studi di Napoli "Federico II" and INFN \\ E-mail: giuliana.galatiena.infn.it
}

OPERA (Oscillation Project with Emulsion tRacking Apparatus) was a long-baseline experiment at the Gran Sasso laboratory (LNGS) designed to search for $v_{\mu} \rightarrow v_{\tau}$ oscillations in appearance mode. OPERA took data from 2008 to 2012 with the CNGS neutrino beam from CERN. The observation of five $v_{\tau}$ candidates allowed assessing the discovery of $v_{\mu} \rightarrow v_{\tau}$ appearance in the CNGS neutrino beam with a significance of $5.1 \sigma$. The data analysis is still ongoing, with the goal of improving the sensitivity to the sterile neutrino search in the $v_{\mu} \rightarrow v_{\tau}$ and $v_{\mu} \rightarrow v_{e}$ appearance channels and oscillation parameters with reduced statistical uncertainties. Current results will be presented and perspectives discussed.

Neutrino Oscillation Workshop

4 - 11 September, 2016

Otranto (Lecce, Italy)

*Speaker.

${ }^{\dagger}$ On behalf of the OPERA Collaboration 


\section{Introduction}

The OPERA experiment [1] has been designed to conclusively prove the existence of $v_{\mu} \rightarrow v_{\tau}$ oscillations. The direct appearance search is based on the detection of $\tau$ leptons produced in $v_{\tau}$ charged current interactions (CC). The challenge of the OPERA experiment to detect the shortlived $\tau$ lepton ( $\tau=87 \mu \mathrm{m}$ ), produced in the CC $v_{\tau}$ interactions, out of almost twenty thousands $v_{\mu}$ interactions, was achieved using the nuclear emulsion technique that features micrometric spatial resolution.

\section{The CNGS beam and the OPERA detector}

The OPERA detector was located in the underground Gran Sasso Laboratory (LNGS), $730 \mathrm{~km}$ away from the neutrino source, in the high energy long-baseline CERN to LNGS beam (CNGS) $[2,3]$. The average neutrino energy was about $17 \mathrm{GeV}$, the $\bar{v}_{\mu}$ contamination $2.1 \%$ in terms of interactions, the $v_{e}$ and $\bar{v}_{e}$ together below $1 \%$, while the number of prompt $v_{\tau}$ negligible. The detector was an hybrid apparatus consisting of an emulsion/lead target complemented by electronic detectors. It was made up of two identical super-modules aligned along the CNGS beam direction, each made of a target section and a muon spectrometer. Each target section consisted of a multi-layer array of 31 target walls interleaved with pairs of planes of plastic scintillator strips. Target walls were made of Emulsion Cloud Chamber target units, called bricks, which were, in total, 150000. Each brick consists of 57 emulsion films, $300 \mu \mathrm{m}$ thick, interleaved with 56 lead plates, $1 \mathrm{~mm}$ thick, for a total mass of $8.3 \mathrm{~kg}$. The electronic detectors were used to identify the brick containing the neutrino interaction, for muon identification and its charge and momentum determination.

\section{Event selection and analysis}

Every brick containing an interaction was extracted from the walls to allow for film development, scanning and for the search for $\tau$ decays, carried out in the emulsion scanning laboratories in Europe and Japan with automated optical microscopes. If a secondary vertex was found, a full kinematic analysis was performed combining the measurements in the nuclear emulsion with data from the electronic detectors. The momentum of charged particles can be measured in emulsions by Multiple Coulomb Scattering [4]. For muons crossing the spectrometers, the momentum is measured with a resolution better than $22 \%$ up to $30 \mathrm{GeV} / \mathrm{c}$, and the charge determined [5].

A first hint of a decay topology is the observation of an impact parameter larger than $10 \mu \mathrm{m}$, defined as the minimum distance between the track and the reconstructed vertex, excluding low momentum tracks. The appearance of the $\tau$ lepton is identified by the detection of its characteristic decay topologies, either in one prong (electron, muon or hadron) or in three prongs. Kinematic selection criteria are then applied according to the decay channel.

\section{4. $v_{\mu} \rightarrow v_{\tau}$ oscillation}

Runs with CNGS neutrinos were successfully carried out from 2008 to 2012, with a total CNGS beam intensity of $17.97 \cdot 10^{19}$ protons on target (p.o.t.). For the present analysis, 5408 
events, fully analysed in the emulsion detectors, are used. Five $v_{\tau}$ candidates have been observed, satisfying the kinematic selection criteria.

The first one consists of a 7-prong neutrino interaction. One of the tracks exhibits a kink topology and the daughter track is identified as a hadron through its interaction [6].

The second one has 2 prongs at primary vertex with production of a short track with flight length of $1.54 \mathrm{~mm}$, associated with $\tau$ lepton, and a longer track identified as a hadron. The $\tau$ lepton decays into 3 prongs, which are identified as hadrons on the basis of momentum-range consistency [7].

In the third $v_{\tau}$ candidate the primary vertex is defined by two tracks and a $\gamma$-ray. One of the tracks is identified as being a hadron based on momentum-range selection criteria, the other one as the $\tau$ lepton decaying into a muon; the decay daughter matches with the muon track reconstructed by the electronic detectors, with a measured momentum of $(2.8 \pm 0.2) \mathrm{GeV} / \mathrm{c}$ and negative charge [8].

The fourth $v_{\tau}$ candidate has a primary vertex defined by four tracks and two $\gamma$-rays. One of the tracks shows a kink topology. Its daughter was followed in the downstream bricks. Momentumrange correlation allows to identify it as a hadron [9].

The fifth $v_{\tau}$ candidate's primary vertex consists of the $\tau$ candidate track, which exhibits a kink topology, a charged particle track, four forward-going and two backward-going nuclear fragments. The daughter track is identified as a hadron from its interaction in the same brick [10]. In the analysed sample, $0.25 \pm 0.05$ background events are expected, coming essentially from charmed events with an undetected primary muon, hadronic re-interactions (for the hadronic decay channels) and large angle muon scattering (for the $\tau \rightarrow \mu$ channel).

Taking into account the different signal-to-noise ratio for each decay channel, the five observed candidates give a $5.1 \sigma$ significance for the exclusion of the background-only hypothesis of $v_{\mu} \rightarrow v_{\tau}$ oscillations [10].

An event with a very peculiar topology was also observed: it has two secondary vertices within about $1 \mathrm{~mm}$ from the primary one. Possible interpretations include $v_{\tau}$ interaction with charm production, or $v \mathrm{NC}$ interaction with double charm production. The proposal considered negligible the observation of events with two secondary vertices and no analysis procedure was designed for such an observation, thus a dedicated analysis was performed. The discrimination was performed using multivariate analysis methods. The event turned out to be very likely a $v_{\tau}$ $\mathrm{CC}$ interaction with charm production. With respect to the non tau-charm hypothesis, the observed event has a very low probability, about $10^{-4}$, which corresponds to a significance of $3.5 \sigma[11,12]$.

\section{5. $v_{\mu} \rightarrow v_{e}$ oscillation}

The tracking capabilities of emulsions also allow to identify electrons produced in CC interactions of $v_{e}$, and therefore to search for $v_{e}$ appearance from $v_{\mu} \rightarrow v_{e}$ oscillations [13]. 34 $v_{e}$ candidate events have been observed. This number is compatible with the expected $v_{e}$ from the beam contamination ( $36.7 \pm 5)$, together with two main sources of background: $\pi^{0}$ misidentified as electron in neutrino interactions without a reconstructed muon and $v_{\tau}$ CC interactions with the decay of the $\tau$ into an electron $(1.2 \pm 0.1)$. Using the following oscillation parameters: $\sin ^{2}\left(2 \theta_{13}\right)=0.098, \sin ^{2} 2 \theta_{23}=1, \Delta m_{32}^{2}=\Delta m_{31}^{2}=2.44 \times 10^{3} \mathrm{eV}^{2}$, assuming $\delta_{C P}=0$ and neglecting matter effects, $2.9 \pm 0.4$ oscillated $v_{e} \mathrm{CC}$ events are expected to be detected in the whole energy range. The number of observed events is compatible with the 3 -flavour oscillation model. 


\section{Sterile neutrino search via $v_{\mu} \rightarrow v_{\tau}$ and $v_{\mu} \rightarrow v_{e}$ oscillations}

OPERA $v_{\tau}$ and $v_{e}$ appearance results have been used to derive limits on the mixing parameters of a massive sterile neutrino [14]. In presence of a fourth sterile neutrino with mass $m_{4}$, the oscillation probability is a function of the $4 \times 4$ mixing matrix $U$ and of the three squared mass differences. Observed neutrino oscillation anomalies, if interpreted in terms of one additional sterile neutrino, suggest $\left|\Delta m_{41}^{2}\right|$ values at the $\mathrm{eV}^{2}$ scale. In the framework of the $3+1$ model, at high values of $\Delta m_{41}^{2}$, the measured $90 \%$ C.L. upper limit on the mixing term $\sin ^{2} 2 \theta_{\mu \tau}=4\left|U_{\mu 4}\right|^{2}\left|U_{\tau 4}\right|^{2}$ is 0.116 , independently of the mass hierarchy of the three standard neutrinos. The OPERA experiment extends the exclusion limits on $\Delta m_{41}^{2}$ in the $v_{\mu} \rightarrow v_{\tau}$ appearance channel down to values of $10^{-2} \mathrm{eV}^{2}$ at large mixing for $\sin ^{2} 2 \theta_{\mu \tau} \gtrsim 0.5$.

Concerning the $v_{\mu} \rightarrow v_{e}$, plot of $\sin ^{2} 2 \theta_{\text {new }}$ was obtained using data collected in 2008 and 2009 [13]. At large $\Delta m^{2}$, the limit on oscillation parameters is $<7.2 \cdot 10^{-3}$ at $90 \% \mathrm{CL}$. This analysis is being updated with full $v_{e}$ sample.

\section{Annual modulation of atmospheric muons}

The annual modulation of the atmospheric muons at the underground Gran Sasso National Laboratory ( $3800 \mathrm{~m}$ w.e.) has been measured. Muons underground arise mostly from the decay of $\pi$ and $K$ produced by the interaction of primary cosmic rays with the nuclei of the upper atmosphere. During summer, air temperature increases and the average gas density decreases. The less dense medium allows a longer mean free path of the mesons and increases the fraction of them that decay to produce muons before their first interaction, so correlation between the muon flux observed underground and the air temperature is expected. The observed modulation has a phase of $(216 \pm 6)$ days. Muon rate fluctuations are shown to be positively correlated with atmospheric temperature, with an effective coefficient $\alpha_{T}=0.93 \pm 0.06$, in good agreement with expectations.

\section{Conclusions and perspectives}

The OPERA experiment has been taking data from 2008 to 2012. Five $v_{\tau}$ candidates have been observed and the non-null observation of $v_{\mu} \rightarrow v_{\tau}$ oscillations has been excluded at 5.1 $\sigma$ [10]. A peculiar event with two secondary vertices has been found. After a dedicated analysis, it turned out to be a $v_{\tau}$ CC interaction with charm production with a significance of $3.5 \sigma$. The observed number of $v_{e}$ interactions is compatible with the $3 v$ model expectations, allowing OPERA to set an upper limit in the parameter space for a non-standard $v_{e}$ appearance. Limits on the mixing parameters of a massive sterile neutrino have also been derived and the exclusion limits on $\Delta m_{41}^{2}$ in the $v_{\mu} \rightarrow v_{\tau}$ appearance channel has been extended down to values of $10^{-2} \mathrm{eV}^{2}$ at large mixing for $\sin ^{2} 2 \theta_{\mu \tau} \gtrsim 0.5$.

The complete OPERA data set is being used also for studying annual modulation of atmospheric muons. Results are in agreement with expectations.

In order to estimate oscillation parameters with reduced statistical uncertainty, an analysis using looser selection cuts and multivariate techniques is being developed. Furthermore, the unique feature of the OPERA experiment to identify all 3 flavours at the same time will be exploited in order to put constraints on the oscillation parameters by doing a joint oscillation fit of all datasets. 


\section{References}

[1] M. Güler et al., An appearance experiment to search for $v_{\mu} \rightarrow v_{\tau}$ oscillations in the CNGS beam: experimental proposal, Tech. Rep. CERN-SPSC-2000-028. LNGS-2000-25. SPSC-P-318, CERN, Geneva, Jul, 2000.

[2] R. Acquafredda et al., The OPERA experiment in the CERN to Gran Sasso neutrino beam, JINST 4 (2009) P04018.

[3] OPERA collaboration, R. Acquafredda et al., First events from the CNGS neutrino beam detected in the OPERA experiment, New J. Phys. 8 (2006) 303, [hep-ex/ 0611023 ].

[4] OPERA collaboration, N. Agafonova et al., Momentum measurement by the Multiple Coulomb Scattering method in the OPERA lead emulsion target, New J. Phys. 14 (2012) 013026, [1106.6211].

[5] OPERA collaboration, N. Agafonova et al., Study of neutrino interactions with the electronic detectors of the OPERA experiment, New J. Phys. 13 (2011) 053051, [11 02 . 1882].

[6] OPERA collaboration, N. Agafonova et al., Observation of a first $v_{\tau}$ candidate in the OPERA experiment in the CNGS beam, Phys. Lett. B691 (2010) 138-145, [1006.1623].

[7] OPERA COLlabORATION collaboration, N. Agafonova et al., New results on $v_{\mu} \rightarrow v_{\tau}$ appearance with the OPERA experiment in the CNGS beam, Journal of High Energy Physics 2013 (2013) 36, [1308.2553].

[8] OPERA collaboration, N. Agafonova et al., Evidence for $v_{\mu} \rightarrow v_{\tau}$ appearance in the CNGS neutrino beam with the OPERA experiment, Phys. Rev. D89 (2014) 051102, [14 01.2079 ].

[9] OPERA collaboration, N. Agafonova et al., Observation of tau neutrino appearance in the CNGS beam with the OPERA experiment, PTEP 2014 (2014) 101C01, [1407. 3513].

[10] OPERA collaboration, N. Agafonova et al., Discovery of $\tau$ Neutrino Appearance in the CNGS Neutrino Beam with the OPERA Experiment, Phys. Rev. Lett. 115 (2015) 121802, [1507. 01417 ].

[11] OPERA collaboration, E. Medinaceli, A neutrino interaction with two vertices topology detected by OPERA, in NuPhys2015: Prospects in Neutrino Physics (NuPhys) London, UK, December 16-18, 2015, 2016. 1604.08249.

[12] OPERA collaboration, M. Roda, Appearance of rare physics phenomena in the OPERA neutrino experiment, PoS NEUTEL2015 (2015) 081.

[13] OPERA COllaboration collaboration, N. Agafonova et al., Search for $v_{\mu} \rightarrow v_{e}$ oscillations with the OPERA experiment in the CNGS beam, JHEP 1307 (2013) 004, [1303. 3953].

[14] OPERA collaboration, N. Agafonova et al., Limits on muon-neutrino to tau-neutrino oscillations induced by a sterile neutrino state obtained by OPERA at the CNGS beam, JHEP 06 (2015) 069, [1503.01876]. 